\title{
Identification of Isolated Organisms from Petro Fields by Gene Sequencing
}

\author{
Preeti Sing \& Ajit Pandya \\ Department of Bio-chemistry, C. U Shah Science College, Ashram road, \\ Ahmedabad \\ Email: ajit1sujit@yahoo.com
}

\begin{abstract}
Hydrocarbon degradation can occur with diverse varieties of microbes in nature. Different chemical compounds have shown their susceptibility to the microbial degradation. . Fungal species generally forms carcinogenic trans - diols while bacterial species generally forms cis - diols. Contaminated soil contained significantly higher amount (50 - $75 \%)$ of gram negative bacteria having genotypes enclosing genes compared to pristine soil ( $0-12.5 \%)$. Application of bioinformatics tools like BLAST has identified microorganism asBacillus cereus, microorganism2 as Pseudoxanthomonasmexicana, microorganism3 as Halomonasdaqingensis and microorganism4 as Parapusillimonasgranuli. These microbes were given names accordingly from PS11 to PS14 respectively. They were assigned unique identification numbers starting from KM192258 to KM192261

Keywords: IDENTIFICATION, GENE SEQUENCING, Bacillus cereus Pseudoxanthomonasmexicana, Halomonasdaqingensis, Parapusillimonasgranuli.
\end{abstract}

\section{Introduction}

Few microbes show versatility for degradation of various compounds while some microbes can degrade only one or two components. $40-80 \%$ of degradation of oil spills is managed by biodegradation process. Marine sediments, soil, estuaries, sea, etc. forms the various habitats for isolation of microbes having hydrocarbon degradation capability. Along with bacteria being the most significant agent for breakdown of hydrocarbons, few of the fungal species like Candida, Fusarium, Trichoderma,

Aspergillus are also known to degrade hydrocarbons. (Adibarata \& Achibana 2009; Omotayo et al. 2011; Kafilzadeh et al. 2010) Amongst bacterial species, few species known for biodegradation of hydrocarbons are Pseudomonas, Acinetobacter, Bacillus, Alcaligens, Micrococcus. $\beta$ - oxidation process is involved in biodegradation of alkanes.(Ting et al. 2009; Joo \& Kim 2013) Aromatic hydrocarbon rings are generally hydroxylated to form diols which form cathecols and subsequently give intermediates of the TCA cycle.

Total degradation of aromatic hydrocarbons produces harmless end products like $\mathrm{CO}_{2}$ and water. Earlier exposure of bacteria to hydrocarbons leads to increased degradation capacity along with the raised population of degrading bacteria at the site of contamination. Bacteria isolated from contaminated sites have greater chances to have plasmid which codes gene responsible for the degradation of hydrocarbon. Few well studies plasmids of Pseudomonas are TOL plasmid for toluene degradation, XYL for xylene degradation, CAM for camphor and SAL for salicylate.

\section{Materials and methods}

There are several methods are available for identification of microbes. Primary method includes study of colony morphology and gram staining. Both these method will give rough idea about the characteristic of microbes. Various Biochemical test as mentioned in the Bergey's manual and MIDI analysis can provide more information about the microbes but ribosomal small subunit sequencing is one of the most efficient and accurate method for identification of living organism. Since sequences of these subunit is highly conserved hence can be used widely for identification of microbes also. 
South American Journal of Academic Research

Special Edition May 2016

As compare to the whole genome these region is very small and unique which makes it more potential for identification. However there are other conserved genes also but rarely used for identification. (Thenmozhi et al. 2011; Kumar et al. 2006; Shukla et al. 2010) Here, 16s rRNA sequencing was done as all the microbes were prokaryotes and the sequences obtained were compare with the database available on NCBI.(Singh \& Fulekar 2010; Mittal \& Singh 2009) All the sequences were submitted to NCBI and given universal identification numbers.

\section{Results}

As mentioned earlier ribosomal RNA gene sequencing was used for the identification of microbes. 16s ribosomal gene sequencing is one of the most reliable method for accurate identification of microbes. (Okoh 2006) The following nucleotide sequences were obtained which were further analyzed using bioinformatics tools.

\section{Microorganism Sequence 1}

gaaaccggggetaataccggataacattttgaaccegcatggttcgaaattgaaaggcggcttcggctgtcacttatggatggaccc gcgtcgcattagctagttggtgaggtaacggctcaccaaggcaacgatgcgtagccgacctgagagggtgatcggccacactggga ctgagacacggeccagactcctacgggaggcagcagtagggaatcttccgcaatggacgaaagtctgacggagcaacgecgcgtg agtgatgaaggctttcgggtcgtaaaactctgttgttagggaagaacaagtgctagttgaataagctggcaccttgacggtacctaacca gaaagccacggctaactacgtgccagcagccgcggtaatacgtaggtggcaagcgttatccggaattattgggcgtaaagcgcgegc aggtggtttcttaagtctgatgtgaaagcccacggctcaaccgtggagggtcattggaaactgggagacttgagtgcagaagaggaaa gtggaattccatgtgtagcggtgaaatgcgtagagatatggaggaacaccagtggegaaggcgactttctggtctgtaactgacactga ggcgcgaaagcgtggggagcaaacaggattagataccetggtagtccacgecgtaaacgatgagtgctaagtgttagagggtttccg ccctttagtgctgaagttaacgcattaagcactccgcctggggagtacggccgcaaggctgaaactcaaaggaattgacgggggecc gcacaagcggtggagcatgtggtttaattcgaagcaacgcgaagaaccttaccaggtcttgacatcctctgaaaaccctagagatagg gcttctccttcgggagcagagtgacaggtggtgcatggttgtcgtcagctcgtgtcgtgagatgttgggttaagtccegcaacgagcgc aaccettgatcttagttgecatcattaagttgggcactctaaggtgactgccggtgacaaaccggaggaaggtggggatgacgtcaaat catcatgccccttatgacctgggetacacacgtgctacaatggacggtacaaagagctgcaagaccgcgaggtggagctaatctcata aaaccgttctcagttcggattgtaggctgcaactcgcctacatgaagctggaatcgctagtaatcgcggatcagcatgccgcggtgaat acgttcccgggccttgtacacaccgcccgtcacaccacgagagtttgtaacacccgaagtcggtgggggtaaccttttttgggagccag $\mathrm{cc} \mathrm{c}$

\section{Microorganism 2}

agtgcggggtaatggeccaccaaggcgacgatcggtagctggtctgagaggatgatcagccacactggaactgagacacggtc cagactcctacgggaggcagcagtggggaatattggacaatgggcgcaagcctgatccagccataccgcgtgggtgaagaaggcct tcgggttgtaaagccettttgttgggaaagaaatcctgtcgattaatactcggtggggatgacggtacccaaagaataagcaccggctaa cttcgtgccagcagccgcggtaatacgaagggtgcaagcgttactcggaattactgggcgtaaagcgtgcgtaggtggtggtttaagtc tgctgtgaaagccctgggctcaacctgggaattgcagtggatactggatcactagagtgtggtagagggatgcggaatttctggtgtag cagtgaaatgcgtagagatcagaaggaacatccgtggcgaaggcggcatcctgggccaacactgacactgaggcacgaaagcgtg gggagcaaacaggattagataccctggtagtccacgecctaaacgatgcgaactggatgttgggtgcaacttggcacccagtatcgaa gctaacgcgttaagttcgecgectggggagtacggtcgcaagactgaaactcaaaggaattgacgggggcccgcacaagcggtgga gtatgtggtttaattcgatgcaacgcgaagaaccttacctggtcttgacatccacggaactttccagagatggattggtgcettcgggaac cgtgagacaggtgctgcatggctgtcgtcagctcgtgtcgtgagatgttgggttaagtccegcaacgagcgcaaccettgtcettagttg ccagcacgtaatggtgggaactctaaggagaccgccggtgacaaaccggaggaaggtggggatgacgtcaagtcatcatggccctt acgaccagggctacacacgtactacaatggtggggacagagggctgcaaacccgcgagggtgagccaatcccagaaaccctatctc agtccggattggagtctgcaactcgactccatgaagtcggaatcgctagtaatcgcagatcagcattgctgcggtgaatacgttcccgg gecttgtacacaccgccegtcacaccatgggagtttgttgcaccagaagcaggtagett

Microorganism 3

ggggaaaccaggctaataccgcatacgtcctacgggagaaagcaggggacctttcgggcettgcgetatcggatgagcctatgt cggattagctggttggtgaggtaatggctcaccaaggcgacgatccgtagctggtctgagaggatgatcagccacatcgggactgag acacggcccgaactcctacgggaggcagcagtggggaatattggacaatgggcgcaagcctgatccagccatgccgcgtgtgtgaa gaaggccctcgggttgtaaagcactttcagtggggaagaaagccttccggttaatacccgggaggagggacatcacccacagaaga 
agcaccggctaactccgtgccagcagccgcggtaatacggagggtgcgagcgttaatcggaattactgggcgtaaagcgcgcgtag gcggcttgataagccggttgtgaaagccccgggctcaacctgggaacggcatccggaactgtcaggctagagtgcaggagaggaa ggtagaattcceggtgtagcggtgaaatgcgtagagatcgggaggaataccagtggcgaaggcggecttctggactgacactgacgc tgaggtgcgaaagcgtgggtagcaaacaggattagataccetggtagtccacgccgtaaacgatgtcgactagccgttgggtccttcg cggactttgtggegcagttaacgcgataagtcgaccgectggggagtacggecgcaaggttaaaactcaaatgaattgacgggggec cgcacaagcggtggagcatgtggtttaattcgatgcaacgcgaagaaccttacctaccettgacatcctgcgaaccettcggagacgaa ggggtgccttcgggaacgcagagacaggtgctgcatggctgtcgtcagctcgtgttgtgaaatgttgggttaagtcccgtaacgagcg caacccttgtccctatttgccagcgattcggtcgggaactctagggagactgccggtgacaaaccggaggaaggtggggacgacgtc aagtcatcatggccettacgggtagggctacacacgtgctacaatggtcagtacaaagggttgcgaacttgcgagagtgagccaatcc cagaaagctgatctcagtccggatcggagtctgcaactcgactccgtgaagtcggaatcgctagtaatcgtgaatcagaatgtcacggt gaatacgttccegggecttgtacacaccgccegtcacaccatgggagtggactgcaccagaagtggttagcctaaccttcgggaggg cgatcaccacgg

\section{Microorganism 4}

gggggataactacgcgaaagcgtggetaataccgcatacgccctacgggggaaagggggggattcttcggaacctctcactattg gagcggccgatatcggattagctagttggtggggtaaaggcctaccaaggcgacgatccgtagctggtttgagaggacgaccagcca cactgggactgagacacggcccagactcctacgggaggcagcagtggggaattttggacaatgggggcaaccetgatccagccatc ccgcgtgtgcgatgaaggccttcgggttgtaaagcacttttggcagggaagaaacaggtctggcgaatacctggactgaatgacggta cetgcagaataagcaccggctaactacgtgccagcagccgcggtaatacgtagggtgcaagcgttaatcggaattactgggcgtaaa gcgtgcgcaggcggttcggaaagaagggtgtgaaatcccagggcttaaccttggaatggcattcttaactaccgggctagagtatgtc agaggggggtagaattccacgtgtagcagtgaaatgcgtagagatgtggaggaataccgatggcgaaggcagccccetgggataat actgacgctcatgcacgaaagcgtggggagcaaacaggattagataccetggtagtccacgccctaaacgatgtcaactagctgttgg ggccttcgggecttagtagcgcagctaacgegtgaagttgaccgectggggagtacggtcgcaagattaaaactcaaaggaattgac ggggacccgcacaagcggtggatgatgtggattaattcgatgcaacgcgaaaaaccttacctacccttgacatgtctggaatcccgaa gagatttgggagtgctcgcaagagaaccggaacacaggtgctgcatggctgtcgtcagctcgtgtcgtgagatgttgggttaagtccc gcaacgagcgcaaccettgtcattagttgctacgaaagggcactctaatgagactgccggtgacaaaccggaggaaggtggggatga cgtcaagtcctcatggeccttatgggtagggcttcacacgtcatacaatggtcgggacagagggtcgccaagccgcgaggcggagec aatcccagaaaccegatcgtagtccggattgcagtctgcaactcgactgcatgaagtcggaatcgetagtaatcgcggatcagcatgtc gegtgatacgt

Application of bioinformatics tools like BLAST has identified microorganism1 as Bacillus cereus, microorganism2 as Pseudoxanthomonasmexicana, microorganism3 as Halomonasdaqingensis and microorganism4 as Parapusillimonasgranuli. Thesemicrobes were given names accordingly from PS11 to PS14 respectively. They were assigned unique identification numbers starting from KM192258 to KM192261

\section{References}

[1.] Adibarata, T.H. \&Achibana, S.T., 2009. Microbial Degradation of Crude Oil by Fungi Pre-Grown on Wood Meal. Interdiscplinary studies on Environmental Chemistry, pp.317-322.

[2.] Joo, M.H. \& Kim, J.Y., 2013. Characteristics of crude oil biodegradation by biosurfatant producing bacterium Bacillus subtilis JK-1. Journal of Korean Society for Applied Biological Chemistry, 59, pp.193-200.

[3.] Kafilzadeh, F., Farhangdoost, M. \&Tahery, Y., 2010. Isolation and identification of phenol degrading bacteria from Lake Parishan and their growth kinetic assay. African journal of Biotechnology, 9(40), pp.6721-6726.

[4.] Mittal, A. \& Singh, P., 2009. Isolation of hydrocarbon degrading bacteria from soils contaminated with crude oil spills. Indian Journal of Experimental Biology, 47, pp.760-765.

[5.] Okoh, A.I., 2006. Biodegradation alternative in the cleanup of petroleum hydrocarbon pollutants. Biotechnology and Molecular Biology, 1(2), pp.38-50.

[6.] Omotayo, A.E. et al., 2011. Degradation of aviation fuel by microorganisms isolated from tropical polluted soils. International Journal Of Biological and Chemical Sciences, 5(2), pp.698-708.

[7.] Shukla, K.P., Singh, N.K. \& Sharma, S., 2010. Bioremediation : Developments, Current Practices and Perspectives. Genetic Engineering and Biotechnology Journal, pp.1-20. 
South American Journal of Academic Research

Special Edition May 2016

[8.] Singh, D. \&Fulekar, M.H., 2010. Benzene bioremediation using cow dung microflora in two phase partitioning bioreactor. Journal of hazardous materials, 175, pp.336-343.

[9.] Ting, S.Y., Tan, H.C. \& Aw, C.S., 2009. Hydrocarbon-degradation by isolate Pseudomonas lundensis UTAR FPE2. Malasian Journal of Microbiology, 5(2), pp.104-108. 